Review Article

\title{
The Effectiveness and Safety of Probiotic Supplements for Psoriasis: A Systematic Review and Meta-Analysis of Randomized Controlled Trials and Preclinical Trials
}

\author{
Liuting Zeng $\mathbb{D}^{1},{ }^{1}$ Ganpeng Yu $\mathbb{D},^{2}$ Yang Wu $\mathbb{D}^{1},{ }^{1}$ Wensa Hao, ${ }^{3}$ and Hua Chen $\mathbb{D}^{1}$ \\ ${ }^{1}$ Department of Rheumatology and Clinical Immunology, Peking Union Medical College Hospital, Chinese Academy of Medical \\ Sciences \& Peking Union Medical College, National Clinical Research Center for Dermatologic and Immunologic Diseases (NCRC- \\ DID), Key Laboratory of Rheumatology and Clinical Immunology, Ministry of Education, Beijing, China \\ ${ }^{2}$ People's Hospital of Ningxiang City, Ningxiang City, Hunan Province, China \\ ${ }^{3}$ Institute of Material Medical, Chinese Academy of Medical Sciences \& Peking Union Medical College Institute of Materia Medica, \\ Beijing, China
}

Correspondence should be addressed to Liuting Zeng; zltab2016@hotmail.com, Ganpeng Yu; yuganpeng.guke@hotmail.com, Yang Wu; yangwu202009@163.com, and Hua Chen; chhuaa332211@163.com

Received 5 July 2021; Revised 5 October 2021; Accepted 15 October 2021; Published 13 December 2021

Academic Editor: Daniele Maria-Ferreira

Copyright (c) 2021 Liuting Zeng et al. This is an open access article distributed under the Creative Commons Attribution License, which permits unrestricted use, distribution, and reproduction in any medium, provided the original work is properly cited.

Background. Patients with psoriasis need long-term medication to control their condition. Recent studies suggest that changing the intestinal flora may be a potential treatment. Methods. The databases were utilized to search the randomized controlled trials (RCTs) and preclinical trials about probiotic supplement in the treatment of psoriasis. The retrieval time is from the establishment of these databases to December 2020. RevMan5.3 was used for the risk assessment of bias and meta-analysis. This systematic review was registered in PROSPERO (CRD42021232756). Results. A total of 3 RCTs involving 164 participants were included. Two RCTs showed that probiotics can improve PASI and thereby improve the condition. For inflammationrelated indicators, only one RCT showed that probiotics can improve the levels of CRP and TNF- $\alpha$ but have no obvious improvement effect on IL6. One RCT demonstrated the total effective rate of probiotics in the treatment of psoriasis. For adverse events, one RCT showed that the incidence of adverse events of probiotic treatment was low. Preclinical studies showed that continuous intervention with oral probiotics can significantly improve the progression of psoriasis and reduce the expression of inflammatory factors. The meta-analysis showed that the PASI between two groups was of no statistical significance (SMD 1.83 [-0.41, 4.07], $P=0.11$ ). Meanwhile, probiotics may improve skin thickness (SMD -5.87 [-11.34, -0.41], $P=0.04)$ in animal model. Conclusion. Prebiotics may have a positive effect on alleviating the clinical symptoms of psoriasis, but a large sample of RCTs is still needed to support its therapeutic effect in psoriasis.

\section{Introduction}

Psoriasis is a chronic inflammatory skin disease, which has a long course and tends to recur easily. The incidence of this disease is mainly young and middle-aged, and it has a greater impact on the physical health and mental status of patients. Epidemiology shows that the global prevalence among adults and children is $2-3 \%$ and $0.5-1 \%$, respectively $[1,2]$, and the prevalence rate reported in the United States in 1996 is 2.6\%
[2]. The clinical manifestations are mainly erythema and scaly, which can be affected by the whole body. The scalp and the extensor side of the limbs are more common, and most of them worsen in winter. The course of the disease is longer, and patients are prone to relapse [3]. At present, patients with psoriasis need long-term medication to control their condition. There are many clinical treatment methods, including narrow-band ultraviolet radiation phototherapy, oral acitretin, Tripterygium wilfordii, methotrexate, 
and cyclosporine. Methotrexate and etanercept are often used in China, and recombinant human interleukin, infliximab, adalimumab, ustekinumab, secukinumab, etc. are also used abroad [4-7]. However, side effects such as gastrointestinal reactions and liver and kidney toxicity of these drugs lead to poor patient compliance. In addition, due to the high cost of some biological agents, patients' access to medicines is restricted. Therefore, it is urgent to explore new supplements and alternative treatment options.

Recent studies suggest that the inflammatory microenvironment formed by bacterial products, intestinal immune cell migration, and systemic cytokine release may cause psoriasis $[8,9]$. The diversity of intestinal microbiota in stool samples of patients with psoriasis is significantly reduced, and a variety of digestive tract bacteria are generally reduced in patients with psoriasis and psoriatic arthritis. Hence, changing the intestinal flora may be a potential treatment $[8,9]$. The current research also shows that it has certain advantages to improve the symptoms and pathological process of psoriasis by adjusting and improving the intestinal microbial community strategy of psoriasis $[10,11]$. Methods to improve the regulation of microbial communities include probiotics, prebiotics, and fecal bacteria transplantation [12-14]. At present, preclinical trials and clinical trials of probiotics for the treatment of psoriasis continue to appear. They showed that probiotics can reduce the psoriasis area and severity index of psoriasis patients, inhibit the inflammation level of psoriasis, regulate immune cells, and regulate the composition of the microbiota [15-17]. However, there are still many deficiencies in related studies, and there is a lack of a summary of the above-mentioned research reports and an evaluation of the level of evidence of the abovementioned studies. Meanwhile, some clinical evidence reports are insufficient in sample size estimation, blind method, and methodological quality evaluation. Therefore, a systematic review of the effectiveness and safety of clinical studies and preclinical studies of probiotics in the treatment of psoriasis is imminent. Therefore, this study hopes to conduct a systematic review of randomized controlled trials (RCTs) of probiotics in the treatment of psoriasis and summarize the clinical effects of probiotics in the treatment of psoriasis, in order to provide guidance for clinical practice and provide reference for future RCTs of probiotics to treat psoriasis.

\section{Materials and Methods}

2.1. Protocol. This systematic review and meta-analysis were conducted strictly in accordance with the protocol registered in PROSPERO (CRD42021232756) and PRISMA-guidelines (see supplementary materials).

2.2. Selection Criteria. For RCTs, they were considered eligible if they met the PICO criteria: (1) participants: the patient was diagnosed as an adult patient with psoriasis by a doctor with sufficient clinical qualifications according to the clinical diagnostic criteria for psoriasis. For psoriasis, there is no limit to first diagnosis or recurrence; (2) intervention: the intervention of the experimental group is probiotic preparations, with no restriction on bacteria species, probiotic con- tent, etc. The intervention of the control group is a placebo or other nonprobiotic intervention methods; (3) outcomes: Psoriasis Area and Severity Index (PASI), inflammationrelated indicators, total effective rate, adverse events; (4) study design: RCTs; and (5) exclusion criteria: non-RCTs, non-adult patients.

For animal experiments, preclinical studies were considered eligible if they met the following criteria: (1) animal population: rats or mice, no restriction on strain, age, and so on; (2) intervention: the intervention of the experimental group is probiotic preparations, with no restriction on bacteria species, probiotic content, etc. The intervention of the control group is blank or other nonprobiotic intervention methods; (3) outcomes: improvement of skin damage, inflammatory immune factors, immune cell composition, etc.; (4) and exclusion criteria: absence of a matched control group and the full text is not available.

2.3. Literature Search Strategy. For clinical trials, we searched the English database (Web of Science, Medline, PubMed, and Embase) and Chinese database (China National Knowledge Infrastructure (CNKI), Wanfang Database, VIP Database for Chinese Technical Periodicals, and China Biology Medicine (CBM)). The retrieval time is from the establishment of these databases to 16 December 2020. In addition, we also searched the Cochrane Library (to Issue 12, 2020) and ClinicalTrials.gov. The search strategy of PubMed and Embase is shown in Table 1 as an example.

For animal experiments, we searched the Web of Science, Medline, PubMed, Embase, CNKI, Wanfang Database, VIP Database for Chinese Technical Periodicals, and China Biology Medicine (CBM). The retrieval time is from the establishment of these databases to 16 December 2020. The search strategy of PubMed and Embase is shown in Table 2 as an example.

2.4. Data Extraction and Risk of Bias Assessment. The selection of literature and data extraction were carried out independently by two researchers according to the data extraction table established in advance. The results will be cross-checked after completing the literature selection and data extraction. If the two researchers have a disagreement, they will discuss whether to include or exclude the literature. If they cannot reach a consistent conclusion, they will discuss with all the researchers to resolve it. The data extraction table includes the name of the first author, publication time, country, scale, intervention measures, and outcomes.

The literature quality evaluation adopts Cochrane Collaboration's risk of bias evaluation standard. It mainly includes the following: (1) random sequence generation method; (2) whether to hide the allocation; (3) blind method; (4) whether the outcome data is complete; (5) whether there is a selective report; and (6) other biases. The quality of the literature was evaluated independently by two researchers. If there is a disagreement, the decision is made through discussion with all researchers.

2.5. Statistical Analysis. The Review Manager 5.3 software was used for statistical analysis. For continuous variables, 
TABle 1: Search strategies for PubMed and Embase.

\begin{tabular}{|c|c|}
\hline PubMed & $\begin{array}{c}\text { Probiotics } \\
\text { AND } \\
\text { (Psoriasis OR Psoriases OR Pustulosis of Palms and } \\
\text { Soles OR Pustulosis Palmaris et Plantaris OR } \\
\text { Palmoplantaris Pustulosis OR Pustular Psoriasis of } \\
\text { Palms and Soles) } \\
\text { AND } \\
\text { (random* controlled trial [pt] OR controlled clinical } \\
\text { trial* [pt] OR randomized [tiab] OR placebo [tiab] OR } \\
\text { drug therapy [sh] OR random* [tiab] OR trial* [tiab] } \\
\text { OR group* [tiab]) } \\
\text { NOT } \\
\text { (animals [mh] NOT humans [mh]) }\end{array}$ \\
\hline EMBASE & $\begin{array}{l}\text { 1. "Probiotics"/exp } \\
\text { 2. "psoriasis"/exp } \\
\text { 3. "Pustulosis of Palms and Soles" } \\
\text { 4. "Pustulosis Palmaris et Plantaris" } \\
\text { 5. "Palmoplantaris Pustulosis" } \\
\text { 6. "Pustular Psoriasis of Palms and Soles" } \\
\text { 7. } 2 \text { or } 3 \text { or } 4 \text { or } 5 \text { or } 6 \\
\text { 8. "randomized controlled trial" } \\
\text { 9. "single blind procedure" or "double blind procedure" } \\
\text { 10. "crossover procedure" } \\
\text { 11. } 8 \text { or } 9 \text { or } 10 \\
\text { 12. } 1 \text { and } 7 \\
\text { 13. } 11 \text { and } 12\end{array}$ \\
\hline
\end{tabular}

Table 2: Search strategies for PubMed and Embase.

\begin{tabular}{ll}
\hline & \multicolumn{1}{c}{$\begin{array}{c}\text { Probiotics } \\
\text { AND }\end{array}$} \\
PubMed & \multicolumn{1}{c}{$\begin{array}{c}\text { (Psoriasis OR Psoriases OR Pustulosis of Palms and } \\
\text { Soles OR Pustulosis Palmaris et Plantaris OR } \\
\text { Palmoplantaris Pustulosis OR Pustular Psoriasis of } \\
\text { Palms and Soles) } \\
\text { NOT }\end{array}$} \\
& \multicolumn{1}{c}{ (humans [mh] NOT animals [mh]) } \\
\hline EMBASE "Probiotics"/exp \\
2. "psoriasis"/exp "Pustulosis Palmaris et Plantaris" \\
3. "Palmoplantaris Pustulosis" \\
6. "Pustular Psoriasis of Palms and Soles" \\
7. 2 or 3 or 4 or 5 or 6 \\
8. 1 and 7 \\
\hline
\end{tabular}

the standard mean difference (SMD) was used to describe the effect size, and the confidence interval (CI) is $95 \%$. The $\chi^{2}$ test was used to analyze the heterogeneity between the results. In the case of low heterogeneity $\left(P>0.1, I^{2}<50 \%\right)$, a fixed effects model analysis was performed. If there is heterogeneity between the studies, a random effects model is used.

\section{Results}

3.1. Results of the Search. The total records identified through database searching and other sources were 49 .
According to the search strategy, a total of 3 articles were obtained through preliminary search. By eliminating duplicate documents, carefully reading the title and abstract, a total of 46 articles were excluded. After carefully reading the full text and comparing the selection criteria, 3 RCTs were screened out and finally included [15-17] (Figure 1).

Regarding preclinical research, we initially retrieved a total of 300 articles from 8 databases. After screening, 3 articles that may meet the conditions were obtained, which were subsequently retained. After reading the full text, three eligible animal model research articles were finally included to analyze the effect of probiotic intervention on psoriasis (Figure 2).

3.2. Description of Included Trials. The 3 RCTs are all from different countries, and the research scale is about 20-90 participants. The publication year of the included RCTs is 20132019. The intervention measures of the 3 RCTs are all probiotics, but the sources of probiotics are different. The details of study characteristics are presented in Table 3.

3.3. Risk of Bias of Included Studies. The summary and graph of risk of bias are shown in Figures 3 and 4 .

3.3.1. Sequence Generation and Allocation Concealment. Only Navarro-López et al. [15] described the method of random sequence generation, which is a computer-generated random sequence. Therefore, it is assessed as low risk of bias. The other two RCTs did not describe the method of random sequence generation and were therefore assessed as unclear risk of bias.

$\mathrm{Lu}$ [17] did not describe whether to use allocation concealment, so it was rated as unclear risk of bias. The remaining two RCTs used similar packaging for the tablets of the test group and the control group. They were considered to have adopted allocation concealment and therefore were assessed as low risk of bias.

3.3.2. Blinding, Incomplete Outcome Data and Selective Reporting. Navarro-López et al. [15] and Lu [17] claimed that they used blinding but did not describe the process of blinding implementation, so they were rated as unclear risk of bias. Groeger et al. [16] described the blinding of both patients and researchers and was therefore rated as low risk of bias.

Although there are missing data in Navarro-López et al. [15], the intention-to-treat analysis was used, so it was rated as low risk of bias. Groeger et al. [16] and Lu [17] did not observe incomplete outcomes, so they were rated as low risk of bias. All RCTs do not have selective reporting and are therefore considered a low risk of bias.

3.3.3. Other Potential Bias. Other sources of bias were not observed in 4 RCTs; therefore, the risks of other bias of the RCTs were low.

3.4. PASI. Two RCTs reported PASI $[15,17]$. Due to their different description methods (Navarro-López et al. use percentage improvement to describe PASI, while Lu directly gives PASI score); they cannot be combined for statistical 


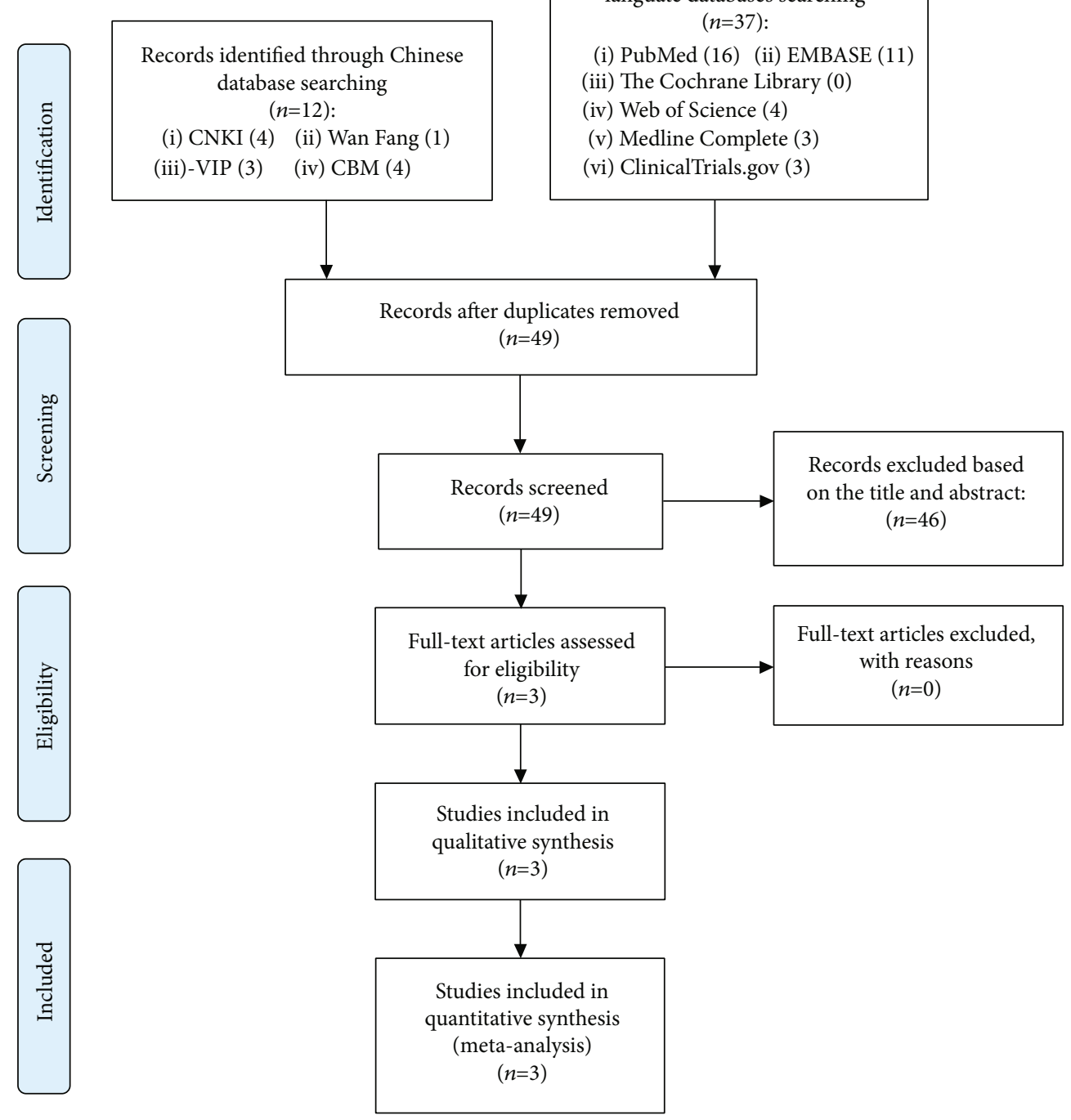

Records identified through other languate databases searching $(n=37)$

(i) PubMed (16) (ii) EMBASE (11)

Figure 1: Flow diagram for searching RCT.

analysis. The results of Navarro-López et al. showed that the improvement of PASI in the probiotic group was better than that in the placebo group $(P=0.03)$. Lu also showed that PASI improved better after probiotic intervention (probiotic vs. placebo: $6.82 \pm 4.22$ vs. $10.76 \pm 5.35 ; P<0.05)$. The results were taken as absolute values and statistically analyzed. The summary results showed that the PASI between two groups was of no statistical significance (SMD 1.83 $[-0.41,4.07], P=0.11$; random effects model) (Figure 5). The funnel chart shows that the possibility of publication bias might be low (Figure S1).

3.5. CRP, TNF- $\alpha$, and IL-6. Only Groeger et al. [16] reported CRP, TNF- $\alpha$, and IL-6. Compared with control group, CRP $(P=0.0425)$ and TNF- $\alpha(P=0.0405)$ were reduced after the intervention of probiotics. For IL-6, the study showed that there was no significant difference between the probiotics intervention and the placebo group $(P>0.05)$.
3.6. Total Effective Rate. Only Lu [17] reported the total effective rate: Total effective rate $=$ the number of cases of (cure + effective + improvement)/total number of cases $x$ $100 \%$. Efficacy index $=($ PASI total score before treatment PASI total score after treatment)/PASI before treatment; cure: curative effect index $>95 \%$; effective: curative effect index 60\%-95\%; improved: curative effect index 30\% $<60 \%$; and ineffective: curative effect index $<30 \%$. Lu showed that in the probiotic group, 14 were cured, 4 were effective, 5 were improved, and 2 were ineffective; the total effective rate was $92 \%$. In the control group, 12 were cured, 4 were effective, 2 were improved, and 7 were ineffective, with a total effective rate of $72 \%$. The difference between the probiotic group and the control group was statistically significant $(P<0.05)$.

3.7. Adverse Events. Only Navarro-López et al. [15] reported adverse events. Navarro-López et al. showed a low incidence 


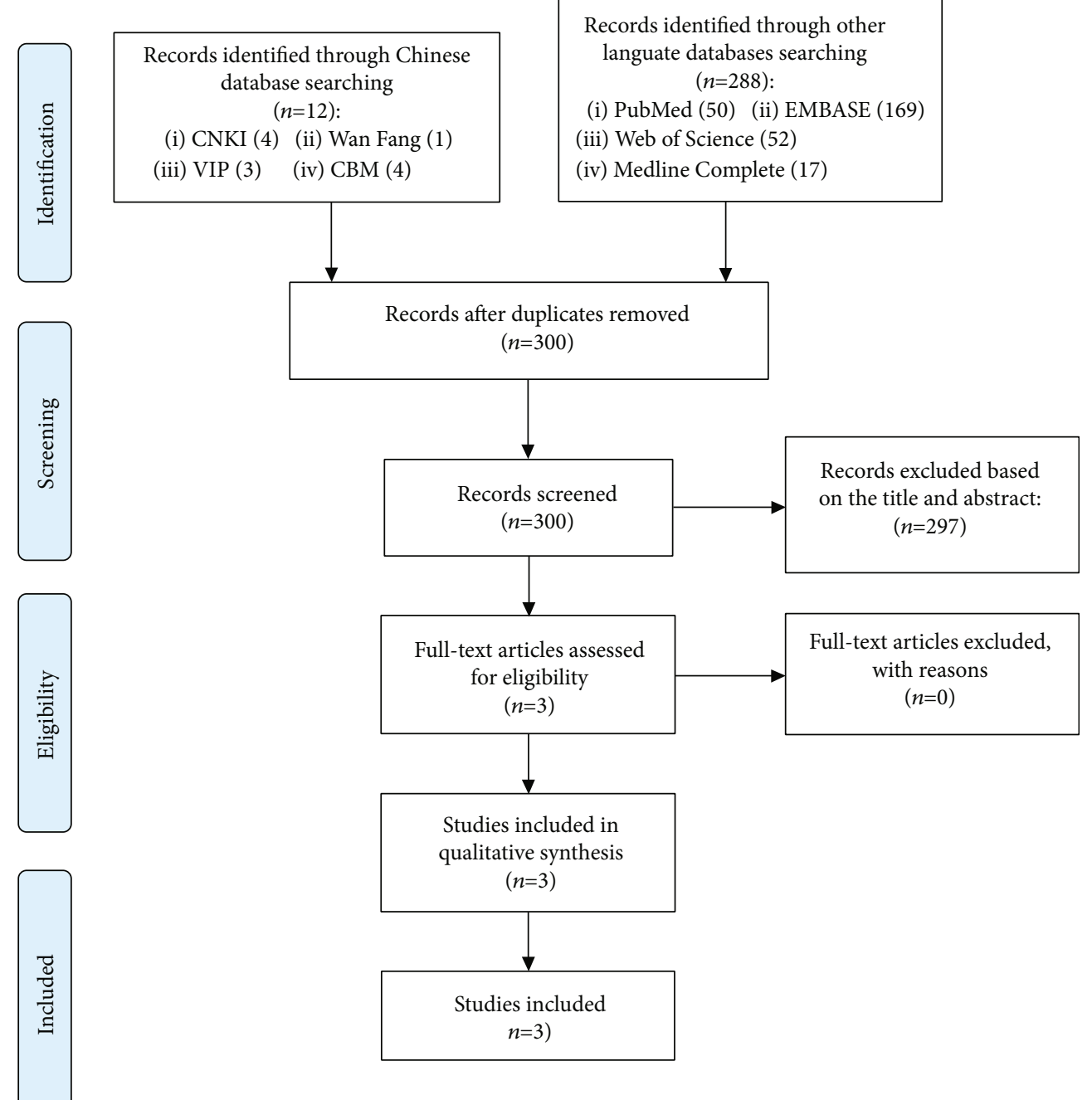

Figure 2: Flow diagram for searching animal experiments.

of adverse events, and no patients withdrew from treatment due to adverse reactions (no specific data shown). It also showed that no serious adverse events occurred in both groups.

3.8. Evidence Quality Assessment. To promote the conclusion, the GRADE tool was utilized to rate the quality of the evidence [18]. According to the GRADE handbook [19], the evidence was judged to be very low (Table 4).

3.9. Animal Studies. This study finally included 3 preclinical studies based on animal models (Table 5). Three studies used different probiotics to treat IMQ-induced psoriasislike skin inflammation model. Chen et al. [20] found that oral administration of $L$. pentosus GMNL-77 may decrease erythematous scaling lesions. It may also decrease the expression of TNF-alpha, IL-6, IL-23, IL-17A/F, and IL-22 mRNA and the number of Th17/Th22 T cells. Rather et al. [21] found that SEL001 may improve skin lesions and pathological changes and decrease vertical skin thickness.
SEL001 may also decrease the expression of IL-19, IL-17A, and IL-23 mRNA. Wang et al. [22] found that Escherichia coli Nissle 1917 improves skin lesions and pathological changes and decreases vertical skin thickness. Escherichia coli Nissle 1917 may decrease the serum IL-8, IL-23, IL-10, and TNF- $\alpha$ levels and increase the serum IL-10 level; it may also decrease the expression of IL-17A, IL-17F, IL-23, TNF- $\alpha$ mRNA, and increase the expression of IL-10 mRNA.

In general, the above-mentioned preclinical animal model studies have shown that continuous intervention with oral probiotics can significantly improve the progression of psoriasis and reduce the expression of inflammatory factors. According to the preliminary results of animal studies, probiotics may have a certain regulatory effect on immune response, inflammatory response, and immune cell composition. The skin thickness of the animal was analyzed statistically. The summary results showed that probiotics can improve skin thickness (SMD -5.87 [-11.34, -0.41], $P=$ 0.04; random effects model), suggesting that it can improve psoriasis skin lesions (Figure 6). 


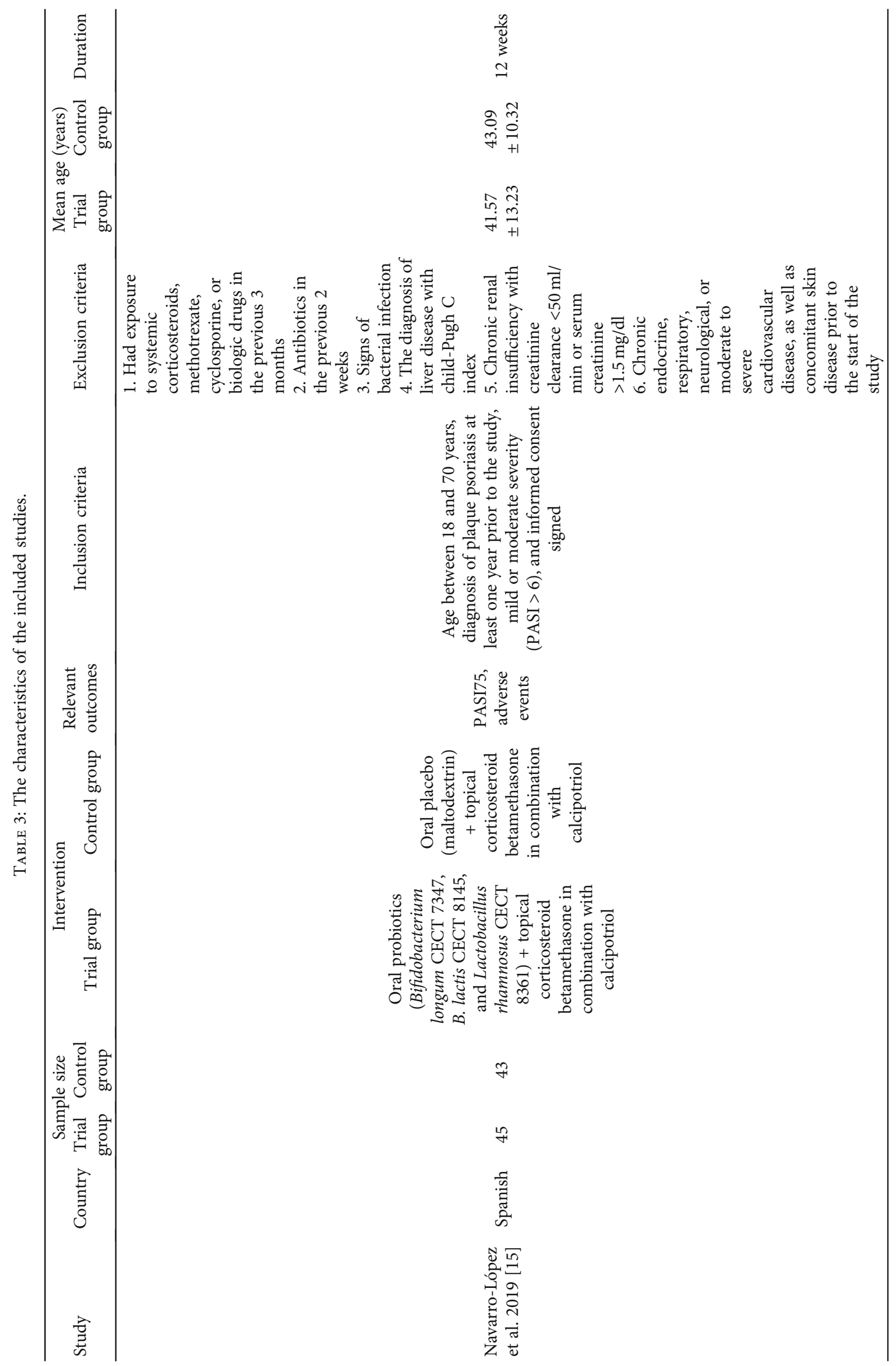




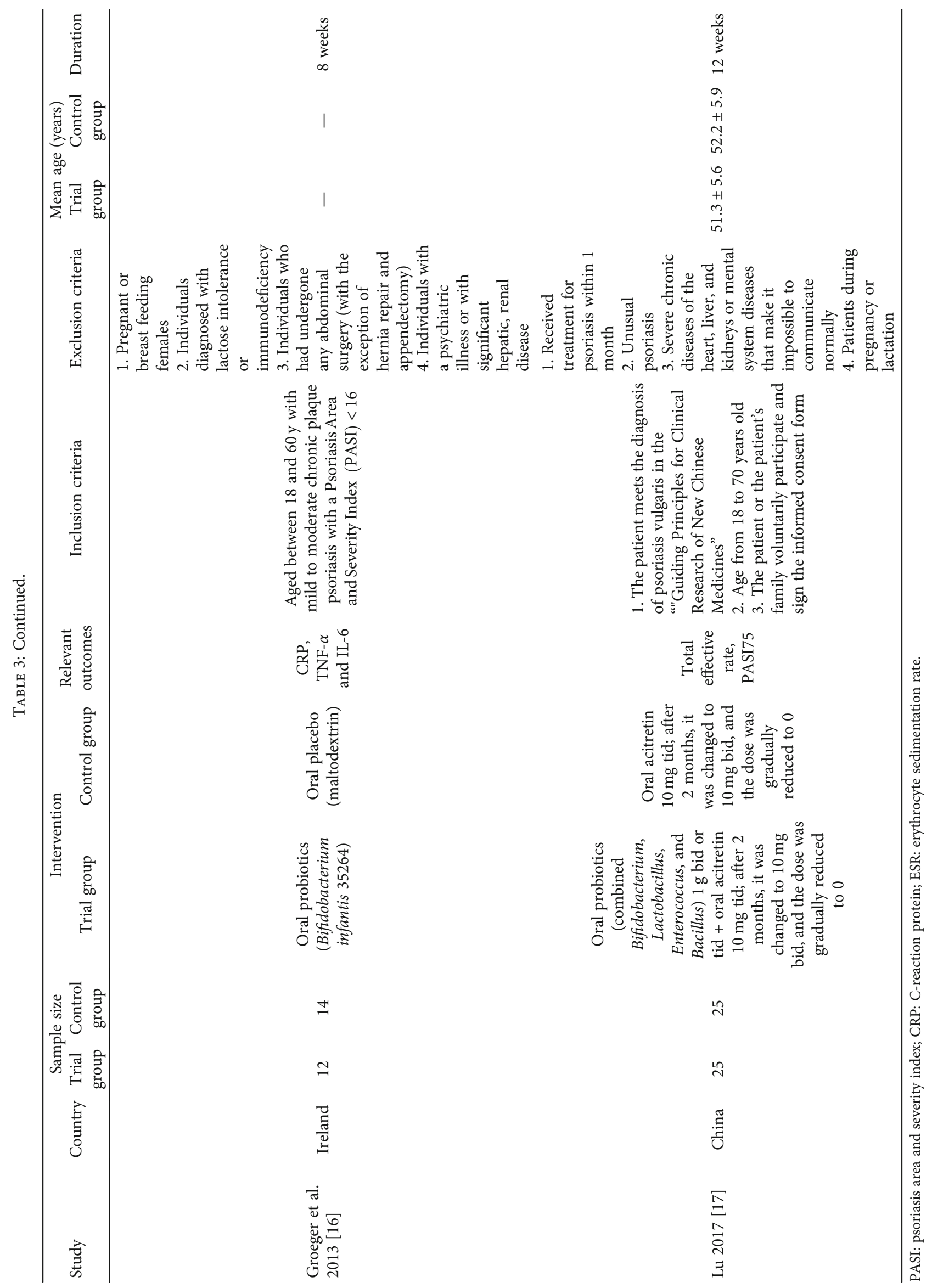




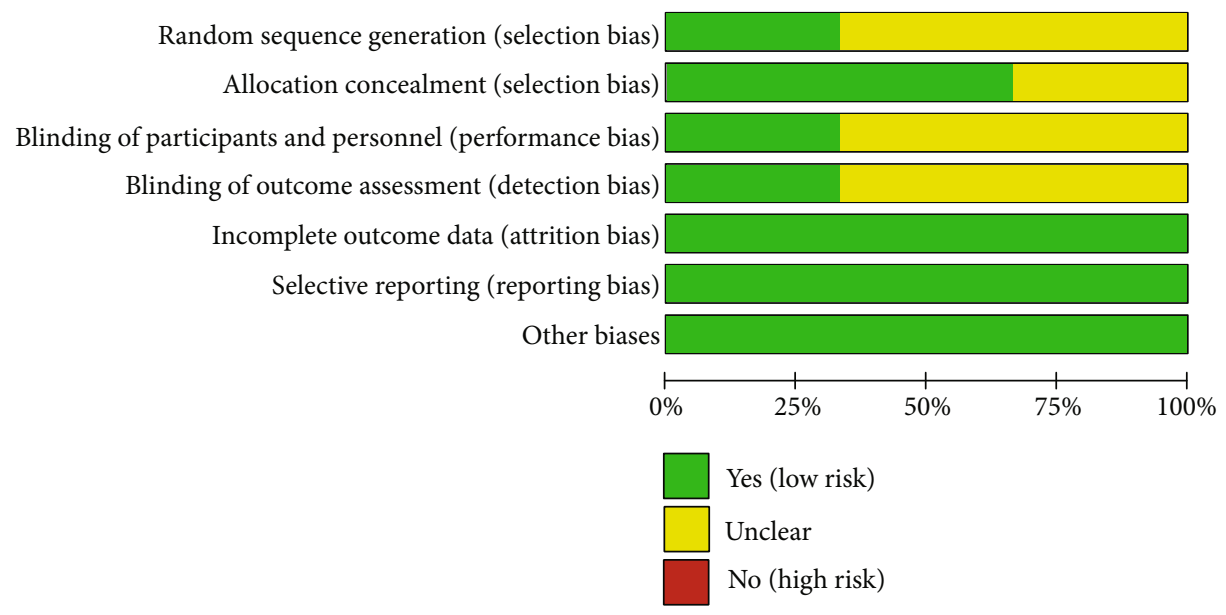

Figure 3: Risk of bias graph.

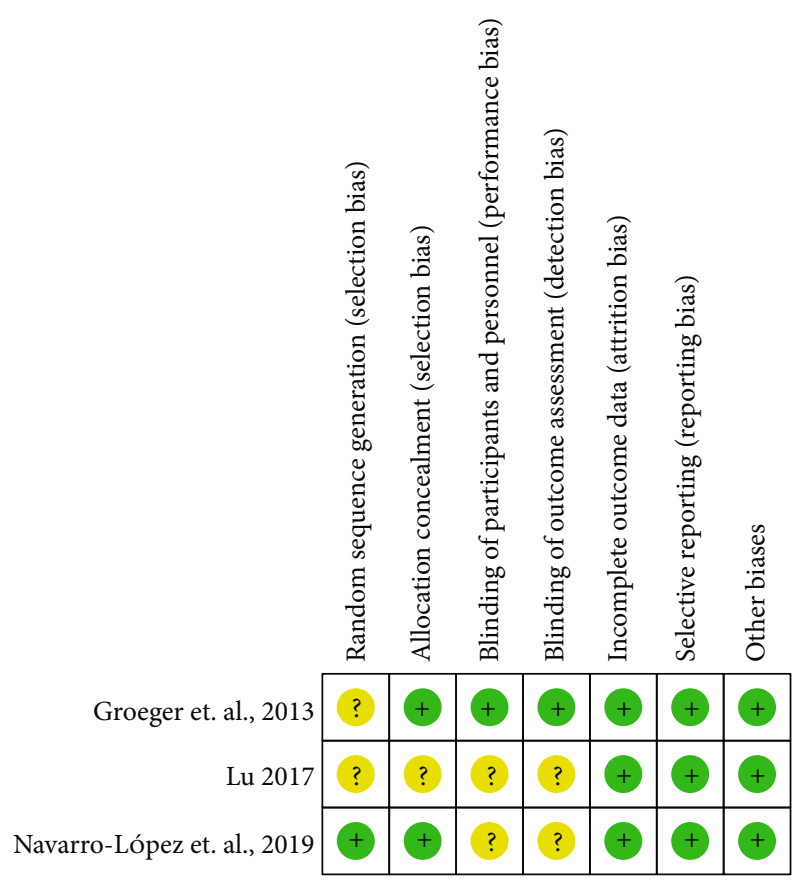

FiguRE 4: Risk of bias summary.

\section{Discussion}

4.1. Main Outcomes Summary. The data of two RCTs reporting PASI supports that probiotics can improve PASI and thereby improve the condition. For inflammationrelated indicators CRP, TNF- $\alpha$, and IL- 6 , only one RCT showed that probiotics can improve the levels of CRP and TNF- $\alpha$ but have no obvious improvement effect on IL6. Lu's study demonstrated the total effective rate of probiotics in the treatment of psoriasis. He defined the treatment result as follows: cure: curative effect index > 95\%; effective: curative effect index 60\%-95\%; improved: curative effect index $30 \% \sim 60 \%$; and ineffective: curative effect index $<30 \%$. Total effective rate $=$ the number of cases of $($ cure + effective + improvement)/total number of cases $\times 100 \%$. Lu showed that in the probiotic group, 14 were cured, 4 were effective,
5 were improved, and 2 were ineffective; the total effective rate was $92 \%$. In the control group, 12 were cured, 4 were effective, 2 were improved, and 7 were ineffective, with a total effective rate of $72 \%$. His research shows that probiotics are more effective in treating psoriasis. Regarding the safety of probiotic therapy, only Navarro-López et al. reported adverse events. Their RCT showed that the incidence of adverse events of probiotic treatment was low, no serious adverse events occurred, and no patients fell off due to adverse events.

4.2. Applicability of Evidences. Psoriasis is a chronic inflammatory skin disease characterized by erythema. The causes of psoriasis are complex, different patients' conditions are quite different, and patients are prone to disease reactions during treatment $[23,24]$. At present, many modern studies believe that a variety of factors, such as patients' genetic factors, infections, metabolic disorders, endocrine disorders, neuropsychiatric factors, and patients' immune disorders, may be the cause of psoriasis [25]. At present, there is no safe, effective, and long-term treatment for psoriasis. In recent years, the incidence of psoriasis has risen sharply, especially among young patients. The results of the psoriasis epidemiological survey report show that in my country, the incidence of psoriasis disease is $0.123 \%$. The treatment of scoria mainly depends on the treatment of drugs such as acitretin or the treatment of narrow-band ultraviolet rays [26]. There are many clinical studies on acitretin, and the results of many studies have confirmed that acitretin has a good effect on psoriasis. However, acitretin may cause a variety of adverse reactions. This results in poor compliance of patients with acitretin, especially in the treatment of young patients with psoriasis [27]. It may cause adverse reactions, such as skin itching, conjunctivitis, neurological symptoms, musculoskeletal pain, and fatigue, and affect the normal work and life of the patient [28]. As a result, the patient cannot take the medication on time according to the doctor's order, and it is easy to cause the patient to stop the drug by himself due to the occurrence of the adverse reaction during the treatment or the patient has to stop the drug treatment due to the serious adverse reaction. In addition, there 


\begin{tabular}{|c|c|c|c|c|c|c|c|c|c|c|c|c|c|}
\hline \multirow{3}{*}{$\frac{\text { Study or subgroup }}{\text { Navarro-López et. al., } 2019}$} & \multicolumn{3}{|c|}{ Experimental } & \multicolumn{2}{|c|}{ Control } & \multirow[b]{2}{*}{ Total } & \multirow[b]{2}{*}{ Weight } & \multirow{2}{*}{$\begin{array}{l}\text { Std. mean difference } \\
\text { IV, random, } 95 \% \text { CI }\end{array}$} & & \multirow{2}{*}{\multicolumn{2}{|c|}{$\begin{array}{l}\text { Std. mean difference } \\
\text { IV, random, } 95 \% \mathrm{CI}\end{array}$}} & \multicolumn{2}{|r|}{ Risk of bias } \\
\hline & Mean & SD & Total & Mean & SD & & & & & & & & 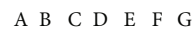 \\
\hline & 0.7 & 0.1 & 45 & 0.4 & 0.1 & 43 & $49.9 \%$ & $2.97[2.36,3.59]$ & & & 드. & & $+(? ?+\oplus+$ \\
\hline Lu 2017 & 12.56 & 5.3 & 25 & 8.38 & 6.52 & 25 & $50.1 \%$ & $0.69[0.12,1.26]$ & & & F & & ? ? ? ? \\
\hline Total $(95 \% \mathrm{CI})$ & & & 70 & & & 68 & $100.0 \%$ & $1.83[-0.41,4.07]$ & & & & & \\
\hline $\begin{array}{l}\text { Heterogeneity: } \text { Tau }^{2}=2.51 ; C \\
\text { Test for overall effect: } Z=1.6\end{array}$ & $\begin{array}{l}=28.39, \mathrm{~d} \\
P=0.11)\end{array}$ & $1(1$ & 0.0000 & 1); $I^{2}=9$ & & & & & $\begin{array}{r}-10 \\
\mathrm{Fa}\end{array}$ & $\begin{array}{l}-5 \\
\text { s (control) }\end{array}$ & $\begin{array}{rr}0 & 5 \\
& \text { Favours }(\mathrm{e}\end{array}$ & $\begin{array}{c}1 \\
10 \\
\text { nental) }\end{array}$ & \\
\hline
\end{tabular}

Risk of bias legend

(A) Random sequence generation (selection bias)

(B) Allocation concealment (selection bias)

(C) Blinding of participants and personnel (performance bias)

(D) Blinding of outcome assessment (detection bias)
(E) Incomplete outcome data (attrition bias)

(F) Selective reporting (reporting bias)

(G) Other biases

FIgURE 5: The results of PASI.

TABLE 4: Summary of findings for the main comparison.

\begin{tabular}{|c|c|c|c|c|}
\hline Outcomes & Illustrative comparative risks $(95 \% \mathrm{CI})^{*}$ & $\begin{array}{l}\text { No of participants } \\
\text { (studies) }\end{array}$ & $\begin{array}{l}\text { Quality of the evidence } \\
\text { (GRADE) }\end{array}$ & Comments \\
\hline PASI & $\begin{array}{l}\text { The mean PASI in the intervention groups was } 1.83 \\
\text { standard deviations higher ( } 0.41 \text { lower to } 4.07 \text { higher) }\end{array}$ & 138 (2 studies) & $\oplus \Theta \Theta \Theta$ very low $w^{1,2,3}$ & SMD $1.83(-0.41$ to 4.07$)$ \\
\hline
\end{tabular}

*The basis for the assumed risk (e.g., the median control group risk across studies) is provided in footnotes. The corresponding risk (and its $95 \%$ confidence interval) is based on the assumed risk in the comparison group and the relative effect of the intervention (and its 95\% CI). CI: confidence interval; GRADE: working group grades of evidence. Very low quality: we are very uncertain about the estimate. ${ }^{1}$ Downgraded one level due to serious risk of bias (random sequence generation, allocation concealment, blinding, and incomplete outcomes), and most of the data comes from the RCTs with moderate risk of bias. ${ }^{2}$ Downgraded one level due to the probably substantial heterogeneity. ${ }^{3}$ Downgraded one level due to the total sample size fails to meet the optimal information size.

are patients who have other health problems caused by taking acitretin, which affects their health. Therefore, the use of acitretin in clinical practice is restricted $[29,30]$. In addition, due to the effects of acitretin on the female reproductive system, acitretin is a contraindicated drug for pregnant women, breastfeeding women, and patients who are planning to have children in the near future [31]. These have also affected the widespread use of acitretin in clinical practice. In addition, due to the occurrence of adverse reactions in the clinic, the dose of acitretin is reduced or the patient can not adhere to the medication, etc., resulting in a significant decrease in the efficacy of acitretin in the treatment of psoriasis [32]. In this case, many patients use acitretin for psoriasis that does not have a good therapeutic effect, or they have repeated attacks. Therefore, the development of safer and more effective treatments in clinical practice is an urgent problem to be solved.

At present, the epidemiological survey of patients with psoriasis and gastrointestinal discomfort has found that the incidence of gastrointestinal discomfort and inflammatory bowel disease in patients with psoriasis is higher than that of healthy people, and vice versa. Another study showed that $7 \%$ to $11 \%$ of patients with inflammatory bowel disease also suffer from psoriasis, which shows that the relationship between psoriasis and gastrointestinal inflammation is particularly close [33-35]. In the study of the intestinal flora of psoriasis and inflammatory bowel disease, it was found that certain beneficial bacteria (such as Clostridium prasectus) were reduced in both psoriasis and inflammatory bowel disease $[8,36,37]$. Meanwhile, patients with psoriasis have significantly increased intestinal inflammation-related auto- antibodies and inflammatory cell infiltration, similar to those in inflammatory bowel disease [38]. Moreover, the difference in intestinal flora is closely related to the host genotype, and there is a certain cross-over between the susceptibility genes of patients with psoriasis and inflammatory bowel disease $[8,39]$. It is inferred from this that psoriasis is related to the systemic inflammatory response and immune problems caused by intestinal flora disorders and that intestinal flora disorders are involved in the systemic lesions associated with psoriasis [8]. On the other hand, certain genetic and environmental factors and immune pathways jointly participate in the pathogenesis of these two diseases. For example, Th17 cells and their cytokines play a major role in the development of psoriasis, and they are also involved in the physiopathological process of inflammatory bowel disease [40]. Intestinal flora can control imiquimodinduced psoriasis skin inflammation by changing $\mathrm{T}$ cell responses, which suggests that intestinal flora affects the pathogenesis of psoriasis [41]. This provides another possible explanation for the relationship between psoriasis, inflammatory bowel disease, and intestinal flora.

Existing studies have found that the diversity and relative abundance of the intestinal flora of patients with psoriasis is significantly reduced [42]. Among them, Bifidobacterium, Broutella, Faecococcus, etc. are significantly reduced in the intestinal tract of patients with psoriasis. Meanwhile, the content of short-chain fatty acid (SCFA) in the stool of patients with psoriasis is significantly lower than that of healthy people [43]. SCFA can regulate the number and functions of $\mathrm{T}$ cell populations by promoting the induction and fitness of $\mathrm{T}$ cells in the colon environment. SCFA 


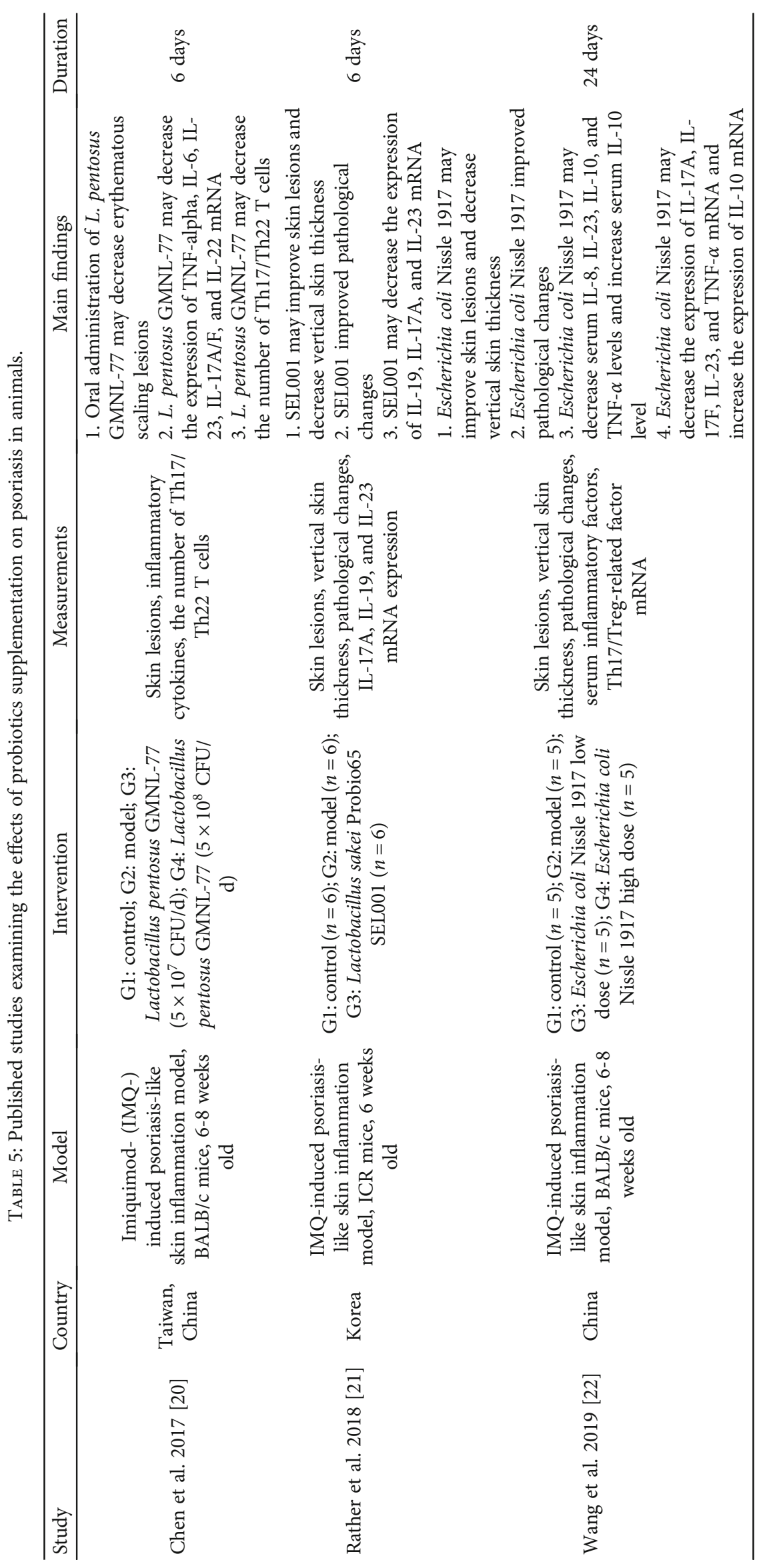




\begin{tabular}{lrrrrrrrr}
\hline \multirow{2}{*}{ Study or subgroup } & Mean & SD & Total & Mean & SD & Total & Weight & Std. mean difference \\
IV, random, 95\% CI
\end{tabular}

Figure 6: Skin thickness.

can play an irreplaceable role in promoting the immune balance of Treg cells such as Th1/Th2 and Th17/Treg. Insufficiency or deficiency of SCFA is related to cellular energy, nutrient metabolism, physical barriers, and immune inflammatory response [44]. Certain species of Firmicutes can upregulate Treg cells through $G$ protein-coupled receptor 43 (GPR43) of SCFA [45]. T/B lymphocytes, DC cells, macrophages, NK cells, and other immune cells are closely related to the pathogenesis of psoriasis [46]. HUANG et al. used 16S rRNA to sequence the feces of 35 patients with psoriasis and 27 healthy people and found that the psoriasis group and the healthy group had differences in flora [47]. The flora of patients with severe psoriasis is different from the flora of patients with mild psoriasis, and the flora of the healthy control group is also different, which confirms that patients with psoriasis have obvious disordered flora [34]. It can be seen that further research on psoriasis based on the microbiota may provide new insights into the pathogenesis of psoriasis and provide more evidence for the prevention and treatment of psoriasis. In addition, studies have found that the diversity of intestinal flora in patients with psoriasis is significantly reduced, and people with low intestinal flora diversity are more likely to be in a lowgrade inflammation state $[48,49]$. Short-chain fatty acids (SCFA) are the fiber fermentation products of the intestinal flora and play a key role in promoting the integrity of the intestinal barrier and exerting anti-inflammatory effects. It is mainly derived from Fischer bacillus, but the content of Fischer bacillus in the intestines of patients with psoriasis is significantly lower than that of healthy subjects $[50,51]$. Butyric acid is an important component of SCFA, which is mainly secreted by Clostridium prastigma of Firmicutes. It is of great significance in promoting the anti-inflammatory effect of SCFA and maintaining the integrity of the intestinal barrier [52]. However, studies have found that the number of Clostridium plasmodium in the intestine of patients with psoriasis has been significantly reduced, suggesting that changes in the composition of the intestinal flora of patients with psoriasis may be an important factor in causing immune inflammation [53]. Another study showed that the increase in the ratio of intestinal Firmicutes/Bacteroidetes in patients with psoriasis is the main feature of the psoriasis intestinal flora, which is specifically manifested in the increase in the abundance of Firmicutes and the decrease in the abundance of Bacteroidetes [54, 55]. An increase in the ratio of Firmicutes/Bacteroidetes can affect the metabolism of intestinal carbohydrates and reduce the production of SCFA, which ultimately leads to chronic inflammation and damage to the intestinal barrier. In addition, impaired intestinal barrier function can directly cause bacteria and their metabolites to be released into the blood and promote the occurrence of systemic inflammatory reactions, thereby inducing or aggravating psoriasis [56]. New research shows that the occurrence and development of psoriasis are closely related to the imbalance of intestinal flora, which can promote the progression of psoriasis by increasing the level of immune inflammatory response in the body. Its core mechanism may be related to the release of intestinal metabolites (LPS and glycolipids) into the blood and activation of inflammatory response and immune-related signaling pathways $[40,57]$.

Probiotics are a class of active microorganisms that produce beneficial effects on the host by regulating the intestinal microecological balance and play an important role in immune regulation, metabolic processes, and neuroendocrine [58]. For a long time, active organisms (probiotics) have been introduced to selectively enhance the intestinal microbiota, or indigestible carbohydrates (prebiotics) have been given to actively promote growth, thereby controlling the intestinal microbiota [59]. The application of prebiotics in atopic dermatitis, acne, and wound healing has achieved good results [60]. Probiotics have an immunomodulatory effect on the skin and enhance the skin barrier repair function by reducing the bacterial load of the skin and antagonizing invasive symbiosis [61]. In a 6-day animal experiment, IRFAN et al. found that Lactobacillus probiotic-65 improved the severity of imiquimod-induced psoriasis in mice. It also reduces the expression levels of psoriasis-related proinflammatory cytokines such as IL-17A, IL-19, and IL-23 [21]. It can be considered that Lactobacillus can not only relieve clinical symptoms but also reduce the level of proinflammatory cytokines. Chen and other teams also confirmed this view. They evaluated the effect of Lactobacillus pentosus GMNL-77 on a mouse model of imiquimod-induced psoriasis. Compared with untreated mice in the control group, mice treated with probiotics had significantly fewer erythema, scales, and thickened epidermis [20]. Magdolna et al. summarized the effects of oral probiotics on psoriasis 
and found that 3 different probiotics have been shown to improve the condition [62]. However, the existing data is limited and uneven, so it is difficult to propose a program for proper supplementation of probiotics in patients with psoriasis.

4.3. Discussion of the Source of Heterogeneity. The heterogeneity of PASI (clinical test results) and skin thickness (animal test results) is high. The high heterogeneity of animal experiment results is mainly due to the different strains, breeding environment, and intervention drugs. The possible reasons for the high heterogeneity of clinical trial outcomes might be as follows: (1) Different RCTs use different probiotic preparations. (2) The populations included in different RCTs are different (the populations involved in the three RCTs are Spanish, Ireland, and China), and there are individual differences. (3) The age groups of patients included in different RCTs are different. (4) Different ways of describing outcomes have caused heterogeneity (Navarro-López et al. use percentage improvement to describe PASI, while Lu directly gives the PASI score).

4.4. The Strengths and Limitation of This Review. The strengths of this systematic review and meta-analysis is that this study evaluated the efficacy and safety of probiotics in the treatment of patients with psoriasis for the first time, comprehensively integrating the RCTs and preclinical experimental results of probiotics in the treatment of patients with psoriasis.

The limitation of this review are as follows: (1) Due to the lack of clinical trials of probiotics for the treatment of psoriasis, this study only included 3 RCTs and 3 preclinical studies, involving only 164 participants, which affected the stability and generalization of the results. (2) The inconsistency of probiotic preparations, different age groups of people, different regions, and different result descriptions have led to the heterogeneity of outcomes. (2) The ambiguity of random sequence generation and allocation, the high heterogeneity of outcomes, and the lack of participants lead to the low quality of evidence (GRADE grading is very low).

4.5. Implications for Future Research. For future clinical practice, the preparation and types of probiotics are recommended to be unified, and it is recommended to report the outcomes in a unified way (such as directly reporting the endpoint PASI score and endpoint inflammation index value). For basic research, it is recommended to further explore the mechanism of probiotics to interfere with psoriasis in the future and explore the effects of probiotics on the intestinal flora and intestinal metabolism of patients with psoriasis. In short, more well-designed and high-quality RCTs and preclinical studies are still needed in the future to correct the results of this study.

\section{Conclusion}

Prebiotics may have a positive effect on alleviating the clinical symptoms of psoriasis and may be used as a treatment strategy for psoriasis, but in the future, a large sample of
RCTs is still needed to support its therapeutic effect in psoriasis.

\section{Abbreviations}

RCT: Randomized controlled trial

CNKI: China National Knowledge Infrastructure

CBM: China Biology Medicine

SCFA: Short-chain fatty acid

GPR43: G protein-coupled receptor 43.

\section{Data Availability}

The data used to support the findings of this study are included within the article.

\section{Conflicts of Interest}

The authors declare no competing interests.

\section{Authors' Contributions}

Ganpeng Yu, Liuting Zeng and Hua Chen are responsible for the study concept and design. Liuting Zeng, Ganpeng $\mathrm{Yu}$, Yang $\mathrm{Wu}$, Wensa Hao, and Hua Chen are responsible for the data collection, data analysis, and interpretation. Liuting Zeng drafted the paper. Hua Chen supervised the study. All authors participated in the analysis and interpretation of data and approved the final paper. We thank Kailin Yang for his help with data collection, data analysis and interpretation, and paper writing. Ganpeng $\mathrm{Yu}$ and Liuting Zeng should be considered joint first author.

\section{Supplementary Materials}

PRISMA 2009 checklist: a checklist of systematic review and meta-analysis for RCT. Figure S1: the funnel chart of PASI. (Supplementary Materials)

\section{References}

[1] C. E. Griffiths and J. N. Barker, "Pathogenesis and clinical features of psoriasis,” The Lancet, vol. 370, no. 9583, pp. 263-271, 2007.

[2] W. H. Boehncke and M. P. Schön, "Psoriasis," The Lancet, vol. 386, no. 9997, pp. 983-994, 2015.

[3] K. Kamiya, M. Kishimoto, J. Sugai, M. Komine, and M. Ohtsuki, "Risk factors for the development of psoriasis," International Journal of Molecular Sciences, vol. 20, no. 18, p. $4347,2019$.

[4] A. Rendon and K. Schäkel, "Psoriasis pathogenesis and treatment," International Journal of Molecular Sciences, vol. 20, no. 6, p. $1475,2019$.

[5] A. W. Armstrong and C. Read, "Pathophysiology, clinical presentation, and treatment of psoriasis: a review," Journal of the American Medical Association, vol. 323, no. 19, pp. 1945-1960, 2020.

[6] A. G. Florek, C. J. Wang, and A. W. Armstrong, "Treatment preferences and treatment satisfaction among psoriasis patients: a systematic review," Archives of Dermatological Research, vol. 310, no. 4, pp. 271-319, 2018. 
[7] M. Tokuyama and T. Mabuchi, "New treatment addressing the pathogenesis of psoriasis," International Journal of Molecular Sciences, vol. 21, no. 20, p. 7488, 2020.

[8] B. Myers, N. Brownstone, V. Reddy et al., "The gut microbiome in psoriasis and psoriatic arthritis," vol. 33, no. 6, p. 101494, 2019.

[9] P. H. Ely, "Is psoriasis a bowel disease? Successful treatment with bile acids and bioflavonoids suggests it is," Clinics in Dermatology, vol. 36, no. 3, pp. 376-389, 2018.

[10] P. Zanvit, J. E. Konkel, X. Jiao et al., "Antibiotics in neonatal life increase murine susceptibility to experimental psoriasis," Nature Communications, vol. 6, no. 1, 2015.

[11] M. Sikora, A. Stec, M. Chrabaszcz et al., "Clinical implications of intestinal barrier damage in psoriasis," Journal of Inflammation Research, vol. 14, pp. 237-243, 2021.

[12] B. Polkowska-Pruszyńska, A. Gerkowicz, and D. Krasowska, "The gut microbiome alterations in allergic and inflammatory skin diseases - an update," Journal of the European Academy of Dermatology and Venereology, vol. 34, no. 3, pp. 455-464, 2020.

[13] M. Sikora, A. Stec, M. Chrabaszcz et al., "Gut microbiome in psoriasis: an updated review," Pathogens, vol. 9, no. 6, p. 463, 2020.

[14] L. Chen, J. Li, W. Zhu et al., "Skin and gut microbiome in psoriasis: gaining insight into the pathophysiology of it and finding novel therapeutic strategies," Frontiers in Microbiology, vol. 11, 2020.

[15] V. Navarro-López, A. Martínez-Andrés, A. Ramírez-Boscá et al., "Efficacy and safety of oral administration of a mixture of probiotic strains in patients with psoriasis: a randomized clinical trial," Acta Dermato Venereologica, vol. 99, no. 12, 2019.

[16] D. Groeger, L. O'Mahony, E. F. Murphy et al., "Bifidobacteriuminfantis 35624 modulates host inflammatory processes beyond the gut," Gut Microbes, vol. 4, no. 4, pp. 325-339, 2013.

[17] X. Y. Lu, "The randomized, double blind and control study of Probiotics in the treatment of psoriasis vulgaris," China Modern Medicine, vol. 24, 2017.

[18] GRADEpro GDT, GRADEpro Guideline Development Tool Software, (2015) McMaster University, 2015, gradepro.org.

[19] H. Schünemann, J. Brożek, G. Guyatt, and A. Oxman, GRADE handbook for grading quality of evidence and strength of recommendations, The GRADE Working Group, 2013, guidelinedevelopment.org/handbook.

[20] Y.-H. Chen, C.-S. Wu, Y.-H. Chao et al., "Lactobacillus pentosus GMNL-77 inhibits skin lesions in imiquimod-induced psoriasis-like mice," Journal of Food and Drug Analysis, vol. 25, no. 3, pp. 559-566, 2017.

[21] I. A. Rather, V. K. Bajpai, Y. S. Huh et al., "Probiotic Lactobacillus sakei proBio-65 extract ameliorates the severity of Imiquimod induced psoriasis-like skin inflammation in a mouse model," Frontiers in Microbiology, vol. 9, 2018.

[22] X. Wang, S. Zhou, F. Yu, X. Su, S. Zhao, and X. Zhu, "Effects of Escherichia coli Nissle 1917 (EcN) on psoriasis mouse model," The Chinese Journal of Dermatovenereology, vol. 33, no. 8, pp. 875-880, 2019.

[23] S. González-Parra and E. Daudén, "Psoriasis and depression: the role of inflammation," Actas Dermo-Sifiliográficas, vol. 110, no. 1, pp. 12-19, 2019.

[24] S. M. Schleicher, "Psoriasis: pathogenesis, assessment, and therapeutic update," Clinics in Podiatric Medicine and Surgery, vol. 33, no. 3, pp. 355-366, 2016.
[25] E. B. Lee, K. K. Wu, M. P. Lee, T. Bhutani, and J. J. Wu, "Psoriasis risk factors and triggers," Cutis, vol. 102, no. 5S, pp. 1820, 2018.

[26] K. M. Hoegler, A. M. John, M. Z. Handler, and R. A. Schwartz, "Generalized pustular psoriasis: a review and update on treatment," Journal of the European Academy of Dermatology and Venereology, vol. 32, no. 10, pp. 1645-1651, 2018.

[27] M. S. Heath, D. R. Sahni, Z. A. Curry, and S. R. Feldman, "Pharmacokinetics of tazarotene and acitretin in psoriasis," Expert Opinion on Drug Metabolism \& Toxicology, vol. 14, no. 9, pp. 919-927, 2018.

[28] T. S. Wang and T. F. Tsai, "Managing scalp psoriasis: an evidence-based review," American Journal of Clinical Dermatology, vol. 18, no. 1, pp. 17-43, 2017.

[29] I. M. G. J. Bronckers, M. M. B. Seyger, D. P. West et al., "Safety of systemic agents for the treatment of pediatric psoriasis," JAMA Dermatology, vol. 153, no. 11, pp. 1147-1157, 2017.

[30] D. M. W. Balak, S. Gerdes, A. Parodi, and L. Salgado-Boquete, "Long-term safety of oral systemic therapies for psoriasis: a comprehensive review of the literature," Dermatology and Therapy, vol. 10, no. 4, pp. 589-613, 2020.

[31] S. B. Kaushik and M. G. Lebwohl, "Psoriasis: which therapy for which patient: focus on special populations and chronic infections," Journal of the American Academy of Dermatology, vol. 80, no. 1, pp. 43-53, 2019.

[32] C. S. Lee and K. Li, "A review of acitretin for the treatment of psoriasis," Expert Opinion on Drug Safety, vol. 8, no. 6, pp. 769-779, 2009.

[33] M. Kim and K. H. Choi, "Inflammatory bowel disease is associated with an increased risk of inflammatory skin diseases: a population-based cross-sectional study," Journal of the American Academy of Dermatology, vol. 76, no. 1, pp. 40-48, 2017.

[34] F. I. Lee, S. V. Bellary, and C. Francis, "Increased occurrence of psoriasis in patients with Crohn's disease and their relatives," AmJGastroenterol, vol. 85, no. 8, pp. 962-963, 1990.

[35] A. D. Cohen, J. Dreiher, and S. Birkenfeld, "Psoriasis associated with ulcerative colitis and Crohn's disease," Journal of the European Academy of Dermatology and Venereology, vol. 23, no. 5, pp. 561-565, 2009.

[36] J. U. Scher, C. Ubeda, A. Artacho et al., "Decreased bacterial diversity characterizes the altered gut microbiota in patients with psoriatic arthritis, resembling dysbiosis in inflammatory bowel disease," Arthritis \& Rhematology, vol. 67, no. 1, pp. 128-139, 2015

[37] L. Tan, S. Zhao, W. Zhu et al., "The Akkermansia muciniphila is a gut microbiota signature in psoriasis," Experimental Dermatology, vol. 27, no. 2, pp. 144-149, 2018.

[38] F. Kerdel and F. Don, "The importance of early treatment in psoriasis and management of disease progression," Journal of Drugs in Dermatology, vol. 17, no. 7, pp. 737-742, 2018.

[39] M. Cottone, C. Sapienza, F. S. Macaluso, and M. Cannizzaro, "Psoriasis and inflammatory bowel disease," Digestive Diseases, vol. 37, no. 6, pp. 451-457, 2018.

[40] M. Komine, "Recent advances in psoriasis research; the clue to mysterious relation to gut microbiome," International Journal of Molecular Sciences, vol. 21, no. 7, p. 2582, 2020.

[41] J. Manasson, D. S. Wallach, G. Guggino et al., "Interleukin-17 inhibition in spondyloarthritis is associated with subclinical gut microbiome perturbations and a distinctive interleukin25-driven intestinal inflammation," Arthritis \& Rhematology, vol. 72, no. 4, pp. 645-657, 2020. 
[42] Z. Zákostelská, J. Málková, K. Klimešová et al., "Intestinal microbiota promotes psoriasis-like skin inflammation by enhancing Th17 response," PLoS One, vol. 11, no. 7, article e0159539, 2016.

[43] B. S. Khyshiktuev, T. M. Karavaeva, and E. V. Fal'ko, "Variability of quantitative changes in short-chain fatty acids in serum and epidermis in psoriasis," Klin Lab Diagn, vol. 8, pp. 2224, 2008.

[44] S. Haase, A. Haghikia, N. Wilck, D. N. Müller, and R. A. Linker, "Impacts of microbiome metabolites on immune regulation and autoimmunity," Immunology, vol. 154, no. 2, pp. 230-238, 2018.

[45] J. Perrigoue, A. Das, and J. R. Mora, "Interplay of nutrients and microbial metabolites in intestinal immune homeostasis: distinct and common mechanisms of immune regulation in the small bowel and colon," Nutrition, Gut Microbiota and Immunity, vol. 79, pp. 57-71, 2014.

[46] Y. Deng, C. Chang, and Q. Lu, "The inflammatory response in psoriasis: a comprehensive review," Clinical Reviews in Allergy and Immunology, vol. 50, no. 3, pp. 377-389, 2016.

[47] L. Huang, R. Gao, N. Yu, Y. Zhu, Y. Ding, and H. Qin, "Dysbiosis of gut microbiota was closely associated with psoriasis," Science China. Life Sciences, vol. 62, no. 6, pp. 807-815, 2019.

[48] S. Omenetti and T. T. Pizarro, "The Treg/Th17 axis: a dynamic balance regulated by the gut microbiome," Frontiers in Immunology, vol. 6, 2015.

[49] E. Le Chatelier, MetaHIT consortium, T. Nielsen et al., "Richness of human gut microbiome correlates with metabolic markers," Nature, vol. 500, no. 7464, pp. 541-546, 2013.

[50] S. Sanna, N. R. van Zuydam, A. Mahajan et al., "Causal relationships among the gut microbiome, short-chain fatty acids and metabolic diseases," Nature Genetics, vol. 51, no. 4, pp. 600-605, 2019.

[51] H. Eppinga, C. J. Sperna Weiland, H. B. Thio et al., "Similar depletion of protective Faecalibacterium prausnitzii in psoriasis and inflammatory bowel disease, but not in hidradenitis suppurativa," Journal of Crohn's and Colitis, vol. 10, no. 9, pp. 1067-1075, 2016.

[52] M. Lopez-Siles, T. M. Khan, S. H. Duncan, H. J. Harmsen, L. J. Garcia-Gil, and H. J. Flint, "Cultured representatives of two major phylogroups of human colonic Faecalibacterium prausnitzii can utilize pectin, uronic acids, and host-derived substrates for growth," Applied and Environmental Microbiology, vol. 78, no. 2, pp. 420-428, 2012.

[53] V. De Preter, K. Machiels, M. Joossens et al., "Faecal metabolite profiling identifies medium-chain fatty acids as discriminating compounds in IBD," Gut, vol. 64, no. 3, pp. 447-458, 2015.

[54] Y. J. Chen, H. J. Ho, C. H. Tseng, Z. L. Lai, J. J. Shieh, and C. Y. $\mathrm{Wu}$, "Intestinal microbiota profiling and predicted metabolic dysregulation in psoriasis patients," Experimental Dermatology, vol. 27, no. 12, pp. 1336-1343, 2018.

[55] C. Hidalgo-Cantabrana, J. Gómez, S. Delgado et al., "Gut microbiota dysbiosis in a cohort of patients with psoriasis," British Journal of Dermatology, vol. 181, no. 6, pp. 12871295, 2019.

[56] R. Corrêa-Oliveira, J. L. Fachi, A. Vieira, F. T. Sato, and M. A. Vinolo, "Regulation of immune cell function by short-chain fatty acids," Clinical \& Translational Immunology, vol. 5, no. 4, p. e73, 2016.
[57] A. Schwarz, R. Philippsen, and T. Schwarz, "Induction of regulatory $\mathrm{T}$ cells and correction of cytokine disbalance by shortchain fatty acids: implications for psoriasis therapy," Journal of Investigative Dermatology, vol. 141, no. 1, pp. 95-104.e2, 2021.

[58] M. Souto-Carneiro, L. Tóth, R. Behnisch et al., "Differences in the serum metabolome and lipidome identify potential biomarkers for seronegative rheumatoid arthritis versus psoriatic arthritis," Annals of the Rheumatic Diseases, vol. 79, no. 4, pp. 499-506, 2020.

[59] C. Maldonado Galdeano, S. I. Cazorla, J. M. Lemme Dumit, E. Vélez, and G. Perdigón, "Beneficial effects of probiotic consumption on the immune system," Annals of Nutrition \& Metabolism, vol. 74, no. 2, pp. 115-124, 2019.

[60] Y.-S. Chang, "Synbiotics for prevention and treatment of atopic dermatitis: a meta-analysis of randomized clinical trials," JAMA Pediatrics, vol. 170, no. 3, pp. 236-242, 2016.

[61] H. Kumar, M. C. Collado, H. Wopereis, S. Salminen, J. Knol, and G. Roeselers, "The bifidogenic effect revisited-ecology and health perspectives of bifidobacterial colonization in early life," Microorganisms., vol. 8, no. 12, p. 1855, 2020.

[62] M. Szántó, A. Dózsa, D. Antal, K. Szabó, L. Kemény, and P. Bai, "Targeting the gut-skin axis-probiotics as new tools for skin disorder management?," Experimental Dermatology, vol. 28, no. 11, pp. 1210-1218, 2019. 